\title{
Lysebehandlung bei Apoplex erfolgreich
}

\author{
Bei Patienten mit akuter zerebraler Ischämie infolge eines intrakraniellen Verschlusses der \\ vorderen Zirkulation ist eine intraarterielle Lysebehandlung effektiv und sicher.
}

\begin{abstract}
_ Die intravenöse Therapie mit Alteplase innerhalb von 4,5 Stunden nach Auftreten der Symptomatik gilt bei Patienten mit akuter zerebraler Ischämie als einzige wirksame Reperfusionstherapie. Kontraindikationen sind allerdings kürzlich erfolgte chirurgische Eingriffe, Gerinnungsstörungen und die Anamnese einer intrakraniellen Blutung.

In 16 niederländischen Kliniken wurde nun die direkte intraarteriellen Reperfusionstherapie untersucht. Rekrutiert wurden 500 Patienten mit akutem Schlaganfall aufgrund eines proximalen arteriellen Verschlusses in der vorderen Hirnzirkulation, bei dem die konventio-
\end{abstract}

nelle Alteplase-Therapie als wenig effektiv gilt. Sie wurde in der Kontrollgruppe durchgeführt. 233 Patienten wurden dagegen in eine Gruppe mit intraarterieller Thrombolyse (Alteplase bis zu $90 \mathrm{mg}$ oder Urokinase bis zu 1,2 Mio. IU) mit oder ohne mechanischer Thrombektomie randomisiert. Ihnen wurde auch ein Stent implantiert.

Nach 90 Tagen ergab sich beim funktionellen Ergebnis eine absolute Differenz von $13,5 \%$ zugunsten der intraarteriellen Therapie (32,6\% vs. 19,1\%). Bei Mortalität oder symptomatischen intrazerebralen Blutungen gab es keine Unterschiede zwischen den Gruppen.
- Berkhemer $O A$ et al. A randomized trial of intraarterial treatment for acute ischemic stroke. N Engl J Med. 2015;372:11-20

\section{KOMMENTAR}

Koronare Interventionen sind längst Routine geworden. Nun erobern Neurologen und Radiologen auch das Gehirn als Feld für interventionelle Gefäßeingriffe. Das Gehirn ist allerdings erheblich anspruchsvoller in seinem Sauerstoff- und Substratbedarf, weswegen das Zeitfenster im Vergleich zum Herzmuskel deutlich kleiner ist. Daraus ergibt sich ein erhöhter logistischer Aufwand für die Versorgung. Aber beim Schlaganfall nur daneben zu stehen und zuzuschauen, ist heute nicht mehr vertretbar.

Prof. Dr. med. H. S. FüeßI

\section{Starb Martin Luther an den Folgen eines akuten Koronarsyndroms?}

Bisher galt der Engländer Edward Earl of Clarendon als Erstbeschreiber eines Angina-pectoris-Anfalls. Er schilderte 1632 die typische Symptomatik, über die sein Vater klagte: „, a sharp pain in the left arm, for a half a quarter of an hour, or near so much, that the torment made him as pale.... as if he were dead". Doch könnte kein geringerer als Martin Luther inm um fast 100 Jahre zuvorgekommen sein!

In einem Brief an Philipp Melanchthon vom 1. Februar 1546 berichtet der Kirchenreformer, was er vier Tage zuvor während eines Spaziergangs bei kaltem Wetter in der Nähe von Unterrißdorf verspürt hatte:

\begin{abstract}
„Auf der Reise befiel mich eine Ohnmacht, und zugleich auch ein Herzanfall. Ich lief nämlich zu Fuß, aber es ging über meine Kräfte, sodass ich schwitzte. Weil danach durch den Schweiß auch das Hemd im Wagen durchkältet war, griff die Kälte einen Muskel des linken Arms an. Daher jene Beklemmung des Herzens und gewissermaßen das Fortbleiben des Atmens. Schuld daran ist meine Torheit, zu Fuß zu gehen. Aber jetzt fühle ich mich wieder ganz leidlich. Wie lange, das weiß ich nicht, weil dem Alter nicht zu trauen ist, zumal auch die Jugend nicht ganz sicher ist."
\end{abstract}

Auf diesen Brief stießen Claudia und Peter Lanzer vom Gesundheitszentrum Bitterfeld/Wolfen in der Ausstellung "Luthers letzte Reise“ im Museum "Luthers Sterbehaus" in Eisleben. Drei Wochen nach dem Spaziergang in der Kälte starb Luther an einer akuten Herzinsuffizienz, der laut Augenzeugen weitere Herzinfarkte vorausgegangen waren.

Dr. med. P. Stiefelhagen

- Lanzer C, LanzerP(planzer@gzbiwo.de).Angina pectoris revisited. Exertional angina preceded Martin Luther's last stretch of a final journey. Eur Heart J. 2016;37:215

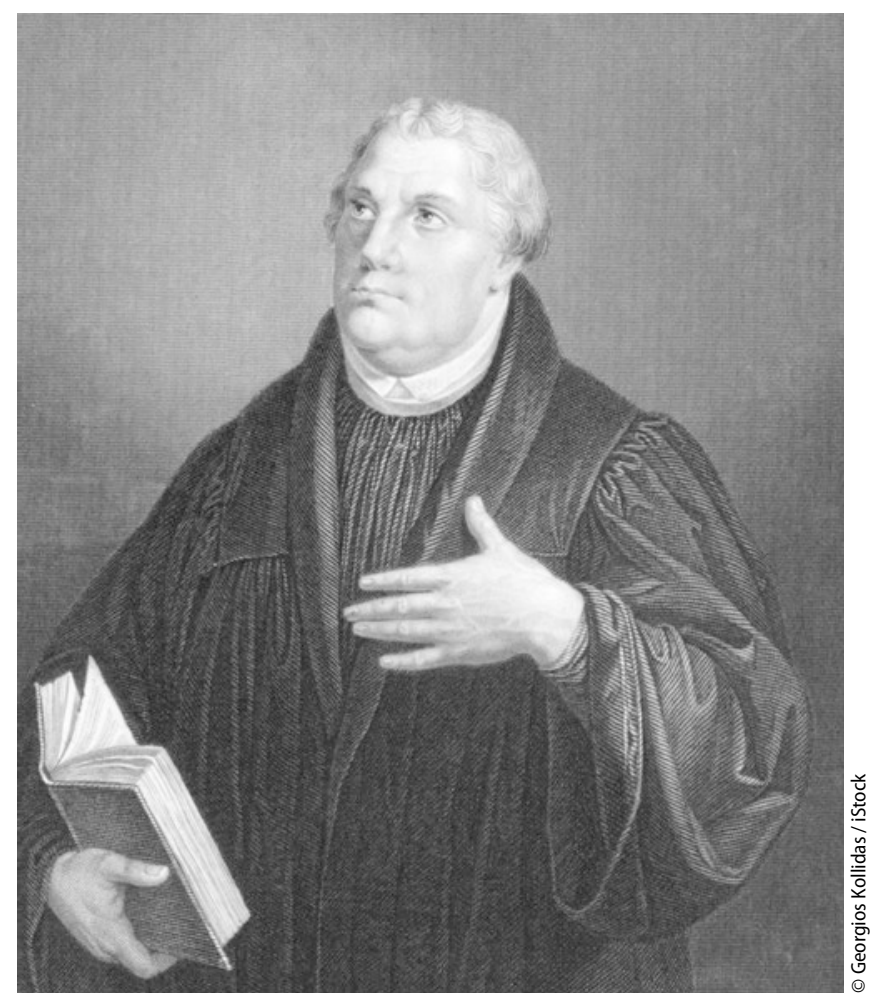

Ereilt den Kirchenreformator hier gerade ein Herzinfarkt? 